\title{
Ist Angst ein guter Ratgeber?
}

\section{Risikoerwartungen als Belastung für den Immobilienmarkt: Das Beispiel des Frackings}

\section{Stephan Heblich}

Online publiziert: 25. Oktober 2019

(C) Der/die Autor(en) 2019

Zusammenfassung Eine transparente Kosten-Nutzen-Rechnung kann dazu beitragen, die Unsicherheit über zukünftige Entwicklungen zu reduzieren und gleichzeitig bietet sie auch einen Richtwert für mögliche Kompensationszahlungen and Eigentümer.

Schlüsselwörter Fracking $\cdot$ Hauspreise $\cdot$ Erwartungen

\section{Fear is not a good advisor}

The detrimental effects of uncertainty on housing markets: the case of fracking

Abstract Robust cost-benefit appraisals of public infrastructures should be made publicly available, in order to avoid uncertainty and increase stability of real estate markets.

Keywords Fracking $\cdot$ House prices $\cdot$ Expectations

\section{Einleitung}

Es wird zunehmend schwer, bauliche Veränderungen gegen die Proteste verschiedener Anspruchsgruppen durchzusetzen. Das betrifft öffentliche Infrastruktur-Projekte - man denke an den Ausbau des Frankfurter Flughafens oder die Waldschlösschenbrücke bei Dresden, städtebauliche Entwicklungsmaßnahmen wie Stuttgart 21, neue Gewerbe, oder auch private Bauvorhaben. Fischel (2001) fasst die Ressentiments ge-

S. Heblich $(\triangle)$

Munk School of Global Affairs \& Public Policy and Department of Economics, University of

Toronto, 1 Devonshire Place, M5S 3K7 Toronto, ON, Kanada

E-Mail: stephan.heblich@utoronto.ca 
gen bauliche Veränderungen, im englischen auch als Locally Unwanted Land Uses (LULU) bezeichnet, passend mit den Akronymen NIMBY (Not In My Backyard) und BANANA (Build Absolutely Nothing Anywhere Near Anybody) zusammen. Die Opposition der Hauseigentümer fußt in der Angst, dass Veränderungen negative Hauspreiseffekte nach sich ziehen könnten und damit zu unmittelbaren Vermögensverlusten führen. Empirisch wird dieser Zusammenhang durch einen Vergleich von Hauseigentümern mit Mietern bei Abstimmungen über Bauvorhaben deutlich sichtbar (siehe Dehring et al. 2008; oder Ahlfeldt und Maennig 2015). Für Hauseigentümer steht mehr auf dem Spiel und folglich setzten sie sich aktiv gegen Vorhaben ein, die den Immobilienwert beeinträchtigen könnten. Erwarten die Eigentümer dagegen eine Steigerung des Immobilienwertes, so unterstützen sie die Vorhaben. Mieter haben letzten Anreiz nicht, da sich die Wertsteigerung im schlimmsten Fall in höheren Mieten widerspiegelt.

Die Widerstände von Hauseigentümern basieren auf Preiserwartungen. Daher ist es wichtig zu verstehen, was diese Erwartungen treibt. Im Folgenden veranschaulicht der Artikel die Erwartungsbildung am Beispiel von Fracking - der Erschließung von Schiefergas - in England (siehe Gibbons et al 2019). Hier führten Ängste vor möglichen Risiken zu deutlichen Immobilienpreisreaktionen. Weitere Untersuchungen legen nahe, dass diese Ängste durch die Medien zusätzlich geschürt wurden. Aus der Sicht politischer Entscheider stellt sich die Frage, wie sich solche (überschießenden) Reaktionen abgemildert lassen, um der negativen Immobilienpreiswirkung und der Ablehnungshaltung entgegenzuwirken. Am Ende des Artikels werden entsprechende Implikationen diskutiert.

\section{Der Fracking-Boom}

Der anhaltende wirtschaftliche Aufschwung in Fracking-Regionen der USA hat weltweit Begehrlichkeiten geweckt. Die technische Möglichkeit, Bohrlöcher in einer Tiefe von bis zu fünf Kilometern horizontal im gashaltigen Schiefergestein zu verzweigen, hat Bohrkosten drastisch gesenkt, da sich Lagerstätten mit einer geringen Anzahl von Bohrungen erschließen lassen. Das hat in den USA einen FrackingBoom ausgelöst und verschiedene Studien deuten auf deutliche Einkommenseffekte hin. In den USA ergeben sich direkte Einkommenseffekte in erster Linie aus Lizenzzahlungen an Grundbesitzer. In anderen Ländern sind die Eigentumsrechte jedoch anders verteilt und es ist wichtig, dies in die Kosten-Nutzen-Rechnung einzubeziehen. In England hält beispielsweise die Krone die Rechte an etwaigen Bodenschätzen unter einem Grundstück und in Deutschland regelt das Bundesbergrecht den Besitz. Demnach gehören grundeigene Bodenschätze wie Dachschiefer, Quarz, oder Basaltlava dem Eigentümer eines Grundstücks. Fossile Brennstoffe gelten dagegen als bergfreie Bodenschätze, die nicht Teil des Eigentums an einem Grundstuck sind. Diese Unterschiede in den Eigentumsrechten haben einen direkten Einfluss auf die lokale wirtschaftliche Bedeutung von Fracking, da sie den Umfang der Zahlungen an Grundbesitzer beeinflussen. Dies wiederum hat Rückwirkungen auf indirekte Multiplikatoreffekte. In den USA zeigen einige Studien, dass gesteigerte Einkommen der lokalen Grundbesitzer und ein florierender Arbeitsmarkt sich 
positiv auf andere Wirtschaftszweige auswirken, weil beispielsweise die Nachfrage nach lokalen Dienstleistungen steigt (Allcott and Keniston 2018; Feyrer et al. 2017). Wenn Grundbesitzer in anderen Ländern geringere und keine Zahlungen erhalten, verringern sich diese Multiplikatoreffekte entsprechend.

Neben den wirtschaftlichen Effekten wird häufig auch darauf verwiesen, dass Erdgas eine umweltverträglichere Alternative zu Kohle und Öl darstellt und die Abhängigkeit von ausländischen Öl- und Gasproduzenten in politisch weniger stabilen Regionen reduziert. Der letzte Punkt ist unbestritten, die Umweltverträglichkeit von Schiefergas ist dagegen nicht eindeutig belegt. Einerseits ist Gas als Brennstoff klimafreundlicher als Kohle oder Öl und kann somit den Übergang zu erneuerbaren Energiequellen unterstützen. Andererseits zeigen Studien, dass bei der Förderung erhebliche Mengen von Methan entweichen können (Howarth et al. 2011). Da Methan um ein Vielfaches klimaschädlicher ist als Kohlendioxid, kann das im schlimmsten Fall die positive Klimabilanz gegenüber Kohle und Öl annullieren.

\section{Negative Externalitäten von Fracking}

Neben den positiven wirtschaftlichen Auswirkungen des Fracking-Booms in den USA, die ihn für andere Länder verlockend erscheinen lassen, gibt es eine zunehmende Zahl von kritischen Studien, die auf negative lokale Externalitäten von Fracking verweisen. Für ein besseres Verständnis dieser Externalitäten ist es hilfreich, den Frackingprozess kurz zu skizzieren. Zunächst wird eine bis zu fünf Kilometer tiefe vertikale Bohrung in das gashaltige Schiefergestein vorgenommen. Davon ausgehend werden mehrere horizontale Bohrungen, sogenannte Laterale, angelegt die den Schaft horizontal verzweigen. Um das Gas aus der gashaltigen Gesteinsschicht typischerweise Schiefergestein - zu lösen, wird ein Gemisch aus Wasser, Quarzsand und Verdickungsmitteln unter einem Druck von bis zu 1000 bar in das horizontale Bohrloch gepresst. Das erzeugt Risse im Schiefergestein die durch die Sandkörnchen offengehalten werden. Auf diese Weise wird das Schiefergas freigesetzt.

In diesem Frackingprozess kann es an verschiedenen Stellen zu negativen Externalitäten kommen. Erstens kann die Bohrung in dicht besiedelten Gebieten zu Lärmbelästigungen führen. Zweitens bedarf Fracking gewöhnlich mehr Wasser, als lokal verfügbar ist. Daher wird Wasser mit Tanklastern angeliefert. Das somit erhöhte Verkehrsaufkommen bedingt wiederum eine gesteigerte Lärmbelästigung, Luftverschmutzung, Straßenabnutzung, und ein höheres Unfallrisiko (Gilman et al. 2013; Muehlenbachs und Krupnick 2014; Colborn und Kwiatkowski 2014; Caulton et al. 2014). Selbst wenn lokale Wasservorkommen ausreichend vorhanden sein sollten, stoppt das nicht den Lastwagenverkehr, denn Frack-Wasser, das als sogenannter Flowback zurückgewonnen wird, ist (in geringerem Masse) durch den Zusatz von chemischen Verdickungsmitteln sowie die natürlich Erdstrahlung, Metalle und Salze kontaminiert und muss in speziellen Einrichtungen gereinigt werden (Olmstead et al. 2013; Warner et al. 2013; Fontenot et al. 2013).

Neben diesen negativen Externalitäten auf Anrainergebiete gibt es zusätzliche Risiken, die Grundbesitzer direkt betreffen können. Eine Studie von Muehlenbachs et al. (2015) in den USA quantifiziert eines der meistdiskutierten Risiken, die mög- 
liche Kontamination von Trinkwasser. Der Film Gasland aus dem Jahr 2010 hat sicherlich dazu beigetragen, Haushalte für das Thema zu sensibilisieren. Josh Fox, der Produzent des Films, dokumentiert die Erfahrungen der Einwohner von Frackingregionen in den USA und stößt dabei häufig auf gesundheitliche Probleme, die im direkten Zusammenhang mit verschmutztem Trinkwasser zu stehen scheinen. Betonummantelungen des Bohrlochs sollen eigentlich verhindern, dass verunreinigtes Frack-Wasser oder Gase in das Grundwasser gelangen. Glauben Hausbesitzer daran, dass die Bohrlochummantelung und umliegende Gesteinsschichten den Austritt von Flowback verhindern, so ist dieses Risiko begrenzt. Gehen Hauseigentümer dagegen davon aus, dass die Betonschicht mit der Zeit porös wird und giftige Flüssigkeiten in das Grundwasser eindringen könnten, so birgt Fracking erhebliche gesundheitliche Risiken. Annahmegemäß sollten Haushalte, die nicht an die öffentliche Wasserversorgung angeschlossen sind und Grundwasser über hauseigene Brunnen nutzen, besonders anfällig für dieses Risiko sein. Die Studie von Muehlenbachs et al. (2015) bestätigt diese Vermutung. Hauspreise in einem Umkreis von $2 \mathrm{~km}$ um eine Frackingquelle steigen aufgrund von Lizenzzahlungen, nachdem die Quellen erschlossen worden ist. Dies gilt jedoch nur für Häuser, die Zugang zur öffentlichen Wasserversorgung haben. Versorgen sich die Haushalte dagegen selbst mit Grundwasser, so zeigt sich ein deutlich negativer Effekt, der den positiven Effekt der Lizenzzahlung überschattet. Diese Ergebnisse unterstreichen, wie wichtig Vertrauen in die Regulierung der Frackingindustrie ist. Hauseigentümer müssen sich darauf verlassen können, dass ausreichende Sicherheitsauflagen vorhanden sind und Verletzungen der Auflagen hart sanktioniert werden. Die Hauspreiseffekte in den USA zeigen, dass Hauseigentümer den Regulierungen nur bedingt vertrauen. In Deutschland äußerte ein Gutachten des Umweltbundesamtes aus dem Jahr 2012 grundlegende Bedenken. Demnach kann nicht ausgeschlossen werden, dass FackFluide irreversible nachteilige Auswirkungen auf die Trinkwasserversorgung haben könnten.

\section{Fracking-induszierte Erdbeben}

Ein zweites Risiko, das Hausbesitzer direkt betrifft, sind seismische Bewegungen. Bislang gibt es noch nicht viele Studien, die einen systematischen Zusammenhang zwischen Fracking und Erdbeben aufzeigen, die Anzeichen verdichten sich aber, dass ein solcher Zusammenhang bestehen könnte (Cheung et al. 2018). Koster und van Ommeren (2015) zeigen am Beispiel der Niederlande, dass konventionelle Erdgasbohrungen (nicht Fracking) seismische Bewegungen auslösen können und diese Erschütterungen spiegeln sich in den Preisen betroffener Häusers wider. Gibbons et al. (2019) finden ähnliche Ergebnisse für Großbritannien. Hier ist kommerzielles Fracking bislang noch nicht erlaubt, die Regierung sieht aber großes Potential und erwägt eine Freigabe in England (in Wales und Schottland haben sich die Lokalparlamente gegen Fracking ausgesprochen). Fracking wurde in Großbritannien erstmalig 2007 in Erwägung gezogen, nachdem geologische Studien große Potentiale versprochen haben. Die Firma Cuadrilla hatte als erstes erfolgreich eine Lizenz beantragt und erhielt die Erlaubnis, eine erste Quelle probehalber zu fracken. Dies 
geschah im Februar 2011, unmittelbar nach dem Fracking kam es jedoch zu leichten seismischen Erschütterungen in der Nähe der Quelle, die von Sensoren aufgegriffen wurden. Daraufhin wurden jegliche weiteren Aktivitäten gestoppt und es folge eine Untersuchung. Da sich aber kein direkter Zusammenhang zum Fracking nachweisen ließ, konnte Cuadrilla im Mai desselben Jahres die Arbeiten fortsetzen. Aber auch der zweite Anlauf führte wieder zu seismischen Bewegungen und dieses Mal wurden weitere Arbeiten bis auf Weiteres unterbunden. Im folgenden Jahr fanden umfangreiche geologische Untersuchungen statt und Gutachten führten die leichten Erdbeben der Stärk 1,7-2,3 auf eine spezielle geologische Struktur zurück. Demnach gab es in der Schieferstruktur eine überdurchschnittlich große Anzahl von Luftblasen, die durch das Fracking platzten und leichte seismischen Bewegungen auslösten. Die Gutachten bewerteten diesen Fall als zufällig und sahen keinen systematischen Zusammenhang zwischen seismischen Bewegungen und Fracking. Auf Basis der Gutachten hatte die Regierung den Frackingbann aufgehoben, neue Projekte stießen aber dennoch auf erheblichen Widerstand in der Bevölkerung und erst im August 2018 wurde ein neues Frackingvorhaben genehmigt.

\section{Medien als Angstverstärker}

Gibbons et al. (2019) quantifizieren den Effekt des Erdbebens auf die Hauspreise in der Region um die Frackingquelle. Die negativen Hauspreiseffekte in Höhe von 3-5\% sind substantiell und übersteigen bei weitem die Höhe der Zahlungen, die den Gemeinden im Frackinggebiet zugesichert wurden. Nähere Untersuchen zeigen, dass der Verfall der Hauspreise kein vorübergehendes Phänomen ist, sondern bis 2015 (dem Ende der Untersuchungsperiode) anhält. Weiter scheint der Effekt regional auf einen Umkreis von ca. 30 Kilometern begrenzt zu sein. Da dieser Umkreis nahezu deckungsgleich mit dem Verbreitungsgebiet lokaler Zeitungen aus der Frackingregion ist, liegt die Vermutung nahe, dass der Effekte wohl zum Teil durch eine Überreaktion auf eine erhöhte Medienberichterstattung zu erklären ist. Nichtsdestotrotz zeigt er, dass Hauseigentümer sensibel auf solche Risiken reagieren. Erdbeben der Stärke 1,7-2,5 haben keine nennenswerten Auswirkungen auf Gebäude, sie lassen aber Raum für Spekulationen, dass es auch zu schwereren Erdbeben kommen könnte und diese Unsicherheit spiegelt sich in den Hauspreiserwartungen wider.

\section{Schlussbemerkungen}

Die Reaktionen auf die durch Fracking ausgelösten seismischen Störungen zeigen, wie Ängste und Erwartungen Hauspreiseffekte beeinflussen können. Oftmals ist es für Investoren schwer, die zu erwartenden Auswirkungen neuer Technologien oder großangelegter baulicher Veränderungen auf die Immobilienpreise einzuschätzen. Und je grösser die Unsicherheit über die zu erwartenden Risiken ist, umso volatiler können die Preisreaktionen ausfallen. Um dem entgegenzuwirken ist es wichtig, die tatsächlich zu erwartenden Kosten und Risiken, sowie den ökonomischen Nutzen und andere positive Effekte verlässlich einzuschätzen und offen zu kommunizieren. 
Eine transparente Kosten-Nutzen-Rechnung kann dazu beitragen, die Unsicherheit über zukünftige Entwicklungen zu reduzieren und gleichzeitig bietet sie auch einen Richtwert für mögliche Kompensationszahlungen and Eigentümer.

Open Access Dieser Artikel wird unter der Creative Commons Namensnennung 4.0 International Lizenz (http://creativecommons.org/licenses/by/4.0/deed.de) veröffentlicht, welche die Nutzung, Vervielfältigung, Bearbeitung, Verbreitung und Wiedergabe in jeglichem Medium und Format erlaubt, sofern Sie den/die ursprünglichen Autor(en) und die Quelle ordnungsgemäß nennen, einen Link zur Creative Commons Lizenz beifügen und angeben, ob Änderungen vorgenommen wurden.

\section{Literatur}

Ahlfeldt GM, Maennig W (2015) Homevoters vs. leasevoters: A spatial analysis of airport effects. J Urban Econ 87(C):85-99

Allcott H, Keniston D (2018) Dutch disease or agglomeration? The local economic effects of natural resource booms in modern America. Rev Econ Stud 85(2):695-731

Caulton DR, Shepson PB, Santoro RL, Sparks JP, Howarth RW, Ingraffea AR, Cambaliza MOL, Sweeney C, Karion A, Davis KJ, Stirm BH, Montzka SA, Miller BR (2014) Toward a better understanding and quantification of methane emissions from shale gas development. Proc Natl Acad Sci 111(17):6237-6242

Cheung R, Wetherell D, Whitaker S (2018) Earthquakes and house prices: evidence from oklahoma. Reg Sci Urban Econ 69(C):153-166

Colborn T, Schultz K, Herrick L, Kwiatkowski C (2014) An exploratory study of air quality near natural gas operations. Hum Ecol Risk Assess Int J 20(1):86-105

Dehring CA, Depken CA, Ward MR (2008) A direct test of the homevoter hypothesis. J Urban Econ 64(1): $155-170$

Feyrer J, Mansur ET, Sacerdote B (2017) Geographic dispersion of economic shocks: Evidence from the fracking revolution. Am Econ Rev 107(4):1313-1334

Fischel WA (2001) The Homevoter Hypothesis: How home values influence local government taxation, school finance and land-use policies. Harvard University Press, Cambridge

Fontenot BE, Hunt LR, Hildenbrand ZL, Carlton DD Jr., Oka H, Walton JL, Hopkins D, Osorio A, Bjorndal B, Hu QH, Schug KA (2013) An evaluation of water quality in private drinking water wells near natural gas extraction sites in the Barnett shale formation. Environ Sci Technol 47(17):10032-10040

Gibbons S, Heblich S, Timmins C (2019) Market tremors: shale gas, earthquakes, and their impact on house prices. Working Paper.

Gilman JB, Lerner BM, Kuster WC, de Gouw JA (2013) Source signature of volatile organic compounds from oil and natural gas operations in north-eastern Colorado. Environ Sci Technol 47(3):1297-1305

Howarth RW, Santoro R, Ingraffea A (2011) Methane and the greenhouse-gas footprint of natural gas from shale formations. Clim Change 106(4):679-690

Koster HR, van Ommeren J (2015) A shaky business: Natural gas extraction, earthquakes and house prices. Eur Econ Rev 80:120-139

Muehlenbachs L, Spiller E, Timmins C (2015) The housing market impacts of shale gas development. Am Econ Rev 105(12):3633-3659

Olmstead SM, Muehlenbachs LA, Shih J-S, Chu Z, Krupnick AJ (2013) Shale gas development impacts on surface water quality in Pennsylvania. Proc Natl Acad Sci 110(13):4962-4967

Warner NR, Christie CA, Jackson RB, Vengosh A (2013) Impacts of shale gas wastewater disposal on water quality in western Pennsylvania. Environ Sci Technol 47(20):11849-11857 